\title{
Sequential Auctions for Common Value Objects with Budget Constrained Bidders
}

\author{
Shaheen S. Fatima \\ Department of Computer Science, Loughborough University \\ Loughborough, LE11 3TU, UK. \\ S.S.Fatima@lboro.ac.uk \\ Michael Wooldridge \\ Department of Computer Science, University of Liverpool \\ Liverpool L69 3BX, UK. \\ mjwaliverpool.ac.uk \\ Nicholas R. Jennings \\ School of Electronics and Computer Science, University of Southampton \\ Southampton SO17 1BJ, UK. \\ nrjeecs.soton.ac.uk
}

\begin{abstract}
This paper analyzes sequential auctions for budget constrained bidders, for multiple heterogeneous common value objects. In most auctions, bidders not only have valuations for objects but also have budget constraints (i.e., constraints on how much they can actually spend in an auction). Given these constraints, the bidders' problem is to determine how much to bid in each auction. To this end, this paper analyzes the strategic behavior of bidders and determines the equilibrium bidding strategies for the individual auctions that constitute a series. We do this in an incomplete information setting where the bidders are uncertain about their budget constraints, and obtain equilibrium bidding strategies for the participants in individual auctions in a series.
\end{abstract}

\section{Introduction}

Negotiation is a key form of interaction in multiagent systems. It is a process in which disputing agents decide how to divide the gains from cooperation. Since this decision is made jointly by the agents themselves [17, 13], each party can only obtain what the other is prepared to allow them. The simplest form of negotiation involves two agents and a single issue. For example, consider a scenario in which a buyer and a seller negotiate on the price of a good. To begin, the two agents are likely to differ on the price at which they believe the trade should take place, but through a process of joint decision-making they either arrive at a price that is mutually acceptable or they fail to reach an agreement. Since agents are likely 
to begin with different prices, one or both of them must move toward the other, through a series of offers and counter offers, in order to obtain a mutually acceptable outcome. However, before the agents can actually perform such negotiations, they must decide the rules for making offers and counter offers. That is, they must set the negotiation protocol or mechanism [17]. On the basis of this protocol, each agent chooses its strategy (i.e., what offers it should make during the course of negotiation). However, in most real-world negotiations, there are more than two parties involved. In such cases, the most widely used mechanisms are auctions.

Auctions are now being widely studied as a means of buying/selling resources in multiagent systems $[1,10,18]$. This is because auctions are not only simple, but can also have desirable economic properties, probably the most important of which are their ability to generate high revenues to the seller and also allocate resources efficiently [20]. Now, in many cases the number of objects to be auctioned is more than one. For example, consider a grid environment where a set of limited processing resources must be sold to a subset of agents that compete for them. Different resources become available for sale at different times and must therefore be sold in sequential auctions.

Fundamentally, there are two approaches to multi-object auctions. The first is to allow bidders to submit bids on bundles of items (these are called combinatorial auctions) [18]. The second is to auction each object independently of all others in a series of auctions (which can either be arranged to run sequentially or in parallel with one another). In this paper we focus on the latter approach and in particular on the sequential case. There are two reasons for this. First, although combinatorial auctions generate economically efficient allocations, they can be computationally complex to implement $[19,18]$. Second, in many real-world auctions where different objects become available at different time points, the objects need to be allocated sequentially.

Given this context, it is important to study the strategic behavior of bidders to determine the equilibrium bidding strategies for such settings. To this end, this paper focuses on sequential auctions for heterogeneous common value objects for budget constrained bidders. Now, in the context of private value auctions, it has been shown that the equilibrium bids for the individual auctions that comprise a series depend on the auction agenda (i.e., the order in which the objects are auctioned) [3]. This ordering is important because, if we change it then the equilibrium bids also change. Given this, we first show that the equilibrium bids for sequential common value auctions also depend on the agenda. Then, we determine the equilibrium bidding strategies for sequential auctions using second-price rules.

In more detail, we study the bidding behaviour of agents for multiple heterogeneous common value objects. Each bidder has a budget constraint which may be different for different bidders. Furthermore, each bidder's budget constraint allows it to buy at most one object. In other words, the scenario is that of multiple objects being sold in a series of sequential auctions and each bidder being able to buy at most one object. Specifically, we determine equilibrium bidding strategies, for each individual auction, for bidders that are uncertain about their budget constraints. This model is an extension of [11], which studied sequential auctions for private value objects with bidders having no budget constraint. Here, we extend this model to common value objects with bidders having budget constraints.

To date, most of the existing work on sequential auctions has focused on scenarios in which all objects are identical $[22,12]$. Sequential auctions for heterogeneous private-value objects have been studied in [16], and those for common value 
objects in [2], but both have been studied under the assumption of complete information. Elmaghraby [8] studies sequential auctions for heterogeneous privatevalue objects using only the second-price sealed-bid rules. Our work differs from the above mentioned work in that we combine the two features of common-values and budget-constraints in our analysis (see Section 5 for details).

Against this background, this paper contributes to the state of the art in multiobject auctions by providing a study of the bidding behaviour of budget constrained bidders for sequential auctions for common-value objects in an incomplete information setting

The remainder of the paper is organized as follows. Section 2 analyzes a complete information model. We then extend the analysis to an incomplete information setting where the bidders are uncertain about each others budget constraints. This setting is described in described in Section 3. For this setting, Section 4 determines equilibrium bids. Section 5 discusses related work and Section 6 concludes.

\section{The Complete Information Setting}

Consider a model where there are two common value objects $(C$ and $D)$ to be auctioned and only two bidders $\left(b_{1}\right.$ and $\left.b_{2}\right)$ that bid for these objects. Bidders $b_{1}$ and $b_{2}$ are constrained not to spend more than $x_{1}$ and $x_{2}$ respectively, where $x_{1}>$ $x_{2}$. We will ignore the trivial case where $x_{1} \geq 2 x_{2}$ (i.e., $b_{1}$ can buy both objects) and concentrate on the situation where each bidder can buy at most one object. Let $a_{1}$ and $a_{2}$ denote the common values of the objects $C$ and $D$ respectively, such that $a_{1}>a_{2}$. For our present analysis, we consider the case where $x_{1} \leq a_{2}$ (i.e., each bidder's budget constraint is less than or equal to the value of each object). As each bidder can buy at most one object, the issue regarding the objects being substitutes or complements does not arise. Let $A_{1}$ denote the auction for the first object and $A_{2}$ that for the second one.

The auction forms we use are as follows. All auctions in the series are conducted using the same form which may be a first- or second-price form. In this section, we consider the complete information setting where each bidder and the auctioneer knows not only the values of the two objects but also each bidder's budget constraint. Given this, we analyze the bidding behaviour and show how this depends on the auction agenda.

\subsection{Second-price auctions}

Consider the case where the second price sealed bid (SPSB) rules are used for auctions $A_{1}$ and $A_{2}$. During the first auction, $A_{1}$, one of the two objects, say $C$, is auctioned. The winner of auction $A_{1}$ is determined and notified. Object $D$ is then auctioned during $A_{2}$. During auction $A_{k}$ (for $k=1,2$ ), bidder $b_{i}$ submits a sealed bid $B_{i}$, and given these bids, the player's payoffs are:

$$
\pi_{i}^{k}= \begin{cases}a_{i}^{k}-\max _{j \neq i} B_{j} & \text { if } B_{i}>\max _{j \neq i} B_{j} \\ 0 & \text { if } B_{i}<\max _{j \neq i} B_{j}\end{cases}
$$

If there is a tie, the object goes to each winning bidder with equal probability.

The problem for a bidder is to find a bidding strategy that will maximize its expected payoff subject to its budget constraint. As we will show, a bidder's payoff for the case where each object is auctioned using the second price sealed bid 
(SPSB) rules is the same as its payoff for the case where each object is auctioned using the English auction rules. To show this, we first analyse each bidders' strategic behaviour for the English auction rules and then for the SPSB rules.

If English auction rules are used for each individual object, the problem for bidders is to determine how much to bid at the first auction. In order to understand this, assume that the first object has been auctioned. The second object will then go to the player who has a higher residual budget (i.e., more money left after the first auction), at a cost equal to the other player's residual budget plus an infinitesimal amount, $\epsilon$. Thus the only decision that each player has to make is how much to bid for the first auction.

Suppose, during auction $A_{1}$, player $b_{2}$ has just bid $B$, which is fractionally more than player $b_{1}$ 's last bid. At this stage player $b_{1}$ needs to decide whether it should continue bidding in the first auction. If player $b_{1}$ decides to bid fractionally more than $B$, and this happens to be the winning bid, it wins the first object and makes a profit of $\left(a_{1}-B\right)$. It seems that if $\left(x_{1}-B>x_{2}\right)$, player $b_{1}$ could also win the second object. But player $b_{2}$ 's equilibrium strategy must be to force player $b_{1}$ up sufficiently, to more than $x_{1}-x_{2}$, so that player $b_{2}$ can get at least one of the two objects. In other words, each bidder exploits the other's budget constraint to maximize its own expected profit. On the other hand, if player $b_{1}$ does not make the next bid during auction $A_{1}$, player $b_{2}$ will get the object at $B$. This will leave player $b_{2}$ with $x_{2}-B$ for the second auction. Since $x_{1}>x_{2}-B$, player $b_{1}$ gets the second object at $x_{2}-B$ and makes a profit of $a_{2}-\left(x_{2}-B\right)$. Thus during the first auction, player $b_{1}$ will bid $B$ provided:

$$
B \leq x_{1} \quad \text { and } \quad\left(a_{1}-B\right)>a_{2}-\left(x_{2}-B\right) .
$$

Let $B_{1}$ denote the highest bid that player $b_{1}$ makes during the auction for the first object. Player $b_{1}$ will continue bidding up to $B_{1}$, where $b_{1}$ satisfies the following constraint:

$$
B_{1} \leq \min \left(x_{1}, \frac{1}{2}\left(a_{1}-a_{2}+x_{2}\right)\right) .
$$

A similar analysis for player $b_{2}$ leads to the criterion that it will continue bidding up to $B_{2}$, where $B_{2}$ satisfies the following condition:

$$
B_{2} \leq \min \left(x_{2}, \frac{1}{2}\left(a_{1}-a_{2}+x_{1}\right)\right) .
$$

Thus, the above two relations give the limits of the bids for the two bidders. An equilibrium outcome will occur when one of the players reaches this limit. The first object will go to the other player at this price. The equilibrium outcome depends on the order in which the two objects are auctioned. This is illustrated in the following example.

Example 1 Let $a_{1}=110, a_{2}=60, x_{1}=60$, and $x_{2}=40$. Let $B_{1}$ denote the bid for player $b_{1}$ and $B_{2}$ that for $b_{2}$ during the auction for the first object. Consider the case where object $C$ is auctioned first. Player $b_{1}$ will bid $B_{1}$ provided the following condition is satisfied.

$$
B_{1} \leq \min \left(60, \frac{1}{2}(110-60+40)\right)=45 .
$$

Player $b_{2}$ will bid $B_{2}$ provided the following condition is satisfied.

$$
B_{2} \leq \min \left(40, \frac{1}{2}(110-60+60)\right)=40 .
$$


Since $B_{1}>B_{2}$, player $b_{1}$ gets the first object for 40 . Thereafter, player $b_{2}$ gets the second object for $60-40=20$. This results in a pay off of 70 to $b_{1}$ and 40 to $b_{2}$. The total revenue to the seller from the two objects is 60 .

Consider the case where the order in which the objects are sold is reversed, i.e., $a_{1}=60$ and $a_{2}=110$. Player $b_{1}$ now bids up to

$$
B_{1} \leq \min \left(60, \frac{1}{2}(60-110+40)\right)=-5 .
$$

Since a player cannot bid negative values, $b_{1}$ does not bid during the first auction. Player $b_{2}$ will bid $b_{2}$ provided

$$
B_{2} \leq \min \left(40, \frac{1}{2}(60-110+60)\right)=5 .
$$

So player $b_{2}$ gets the first object for nothing and player $b_{1}$ gets the second object for 40. This results in a pay off of 60 to both players. The seller's total revenue now is 40 .

As the above example illustrates, the equilibrium outcome for sequential English auctions depends on the order in which the objects are auctioned.

We now turn to the case where the SPSB rules are used for each object. Let $P$ denote:

$$
P=\min \left(x_{1}, \frac{1}{2}\left(a_{1}-a_{2}+x_{2}\right)\right)
$$

and $Q$ denote:

$$
Q=\min \left(x_{2}, \frac{1}{2}\left(a_{1}-a_{2}+x_{1}\right)\right)
$$

During auction $A_{1}$, it is a dominant strategy for bidder $b_{1}$ to bid $B_{1}=P$, and for bidder $b_{2}$ to bid $B_{2}=Q$. In order to understand this consider bidder $b_{1}$. By bidding $B_{1}, b_{1}$ will win if $P>B_{2}$ and lose if $P<B_{2}$ (if $P=B_{2}, b_{1}$ is indifferent between winning and losing). Suppose that $b_{1}$ bids $z_{1}<x_{1}$. If $P>z_{1}>B_{2}$, then $b_{1}$ still wins and its profit is still $a_{1}-P$. On the other hand, if $B_{2}>P>z_{1}$, it still loses. However, if $P>B_{2}>z_{1}$, then it loses whereas if it had bid $P$, it would have made a positive profit. Thus, bidding less than $P$ can never increase $b_{1}$ 's profit but in some cases may actually decrease it. A similar argument can be used to show that it is not profitable for $b_{1}$ to bid more than $P$. In the same way it can be verified that it is a dominant strategy for bidder $b_{2}$ to bid $Q$.

Furthermore, since there are no more objects to be auctioned after $A_{2}$, each bidder's optimal strategy during $A_{2}$ is the same as that for a single object SPSB auction. In other words, the bidder with the lower residual budget bids its budget, and the bidder with the higher residual budget bids infinitesimally more than the other's residual budget. Thus, the outcome of auction $A_{1}\left(A_{2}\right)$, if the English auction rules are used for $A_{1}$ and $A_{2}$ is the same as the outcome of $A_{1}\left(A_{2}\right)$ if the SPSB rules are used for $A_{1}$ and $A_{2}$. In other words, each bidders' payment for auction $A_{1}\left(A_{2}\right)$, if the English auction rules are used is the same as its payment for auction $A_{1}\left(A_{2}\right)$ if the SPSB rules are used.

\section{The Incomplete Information Setting}

There are $m$ common value objects denoted $(1,2, \ldots, m)$ for sale. Each object is sold in a separate auction using the first- or second-price rules and the auctions 
are held sequentially. There are $n$ risk neutral bidders where $n>m-$ the case with $n<=m$ is trivial as each bidder can win an object. The bidders are denoted $b_{1}, b_{2}, \ldots, b_{n}$.

As in Section 2, each bidder has a budget constraint. However, unlike the complete information setting of Section 2, here the information about budget constraints is uncertain to the bidders. Each bidder has a constraint which allows it to buy at most one object. Also, since the sequential auctions are held over a period of time, a bidder's budget may vary over time. We model this variation as follows.

Each bidder's budget constraint is i.i.d in the interval $[0, \omega]$ according to an increasing distribution function $F$. Thus, each bidder's budget constraint is a random variable denoted $X$. The probability distribution function $F$ for $X$ is known to the bidders. However, each bidder draws its budget from $F$. If $x_{i}$ denotes $b_{i}$ 's realization of $X$, then $x_{i}$ is known only to $b_{i}$, but not to other bidders.

Assume that the seller has access to a private source of information ${ }^{1}$ about the value of the two objects. Since each player is a profit maximizer, and for common value auctions, it has been shown that an auctioneerr can raise its revenue by revealing its information publicly [14], we consider the case where the auctioneer makes its private information known to all bidders before the auctions begin. The seller and all $n$ bidders thus know the values, $a_{1}, \ldots, a_{m}$, of the $m$ objects. The objects have additive value (i.e., they are neither substitutes nor complements). These objects are auctioned using $m$ auctions $\left(A_{1} \ldots, A_{m}\right)$ that are carried out sequentially, one after another. The agenda is known to the auctioneer and the bidders. As in the case of the complete information setting, the winner for auction $A_{i}$ (where $1 \leq i \leq m$ ) is announced and then auction $A_{i+1}$ is conducted.

The sequential auctions are conducted as follows. The first object is sold in $A_{1}$. There are $n$ bidders for this auction. The winning bid for the first auction is announced at the end of the auction. Each bidder needs a single object. Thus the winning bidder for an auction does not participate in any of the subsequent auctions. All the losing bidders for an auction go to the next auction. This process repeats for each of the $m$ objects. In other words, the bidders continue to bid in the auctions only until they win an object. Thus, if there are $n$ bidders in the first auction, then there are $n-1$ bidders for the second, $n-2$ for the third, and so on. In general, there are $n-j+1$ bidders for auction $j(1 \leq j \leq m)$.

In more detail, the auctions are held as follows:

1. All the bidders draw their budget constraints for auction $A_{j}$ from the p.d.f. $F$.

2. Auction $A_{j}$ is held; at the end of the auction, the object is allocated to the winning bidder.

3. The winning bidder for auction $A_{j}$ leaves (because each bidder has unit demand) and the remaining bidders go to the next auction $\left(A_{j+1}\right)$.

4. For the next auction $\left(A_{j+1}\right)$, the bidders draw their budget constraints (constraints for auction $A_{j}(1 \leq j \leq m)$ are drawn from the p.d.f. $\left.F\right)$.

5. Steps 2 to 4 are repeated for each of the remaining objects.

Note that each of the $m$ auctions are held using the same rule which may be English, SPSB, Dutch, or FPSB. For instance, if $A_{1}$ is held using English auction

\footnotetext{
${ }^{1}$ For example, in the context of auctions for oil drilling rights, the auctioneer (i.e., government) may have information about the common value of these rights.
} 
rules, then $A_{2}$ to $A_{m}$ are also held using English auction rules. Likewise, for the other three rules.

For this model we now determine the equilibrium bidding strategies.

\section{Equilibrium Bids for Incomplete Information}

In this setting, the p.d.f for the bidders' budget constraints (i.e., $F$ ), the number of objects $(m)$, the number of bidders for the first auction $(n)$, and the auction agenda are common knowledge to the bidders.

Since there is more than one auction, a bidder's bid for an auction depends not only on that auction but also on the profit he expects to get from the future auctions. This profit depends on the number of bidders that participate in the future auctions. Given this, we first determine this profit and then find the equilibrium bids.

If the number of bidders for the first auction is $n$, then let $\beta_{1}(y, j, m, n)$ denote a bidder's ex-ante probability of winning the $y$ th (for $j \leq y \leq m$ ) auction in the series from the $j$ th to the $m$ th one before the $j$ th auction begins. For instance, consider $\beta_{1}(1,1, m, n)$, which is the probability of winning the first auction in the series of auctions from the first to the $m$ th one. Since $\beta_{1}(1,1, m, n)$ is the ex-ante probability (i.e., before the bidders draw their values for the first auction), each bidder has equal chances of winning the first auction, i.e.,

$$
\beta_{1}(1,1, m, n)=1 / n
$$

Recall that if a bidder wins the first auction, he does not participate in the remaining ones.

Now consider the ex-ante probability $\beta_{1}(2,1, m, n)$, which is the probability that a bidder wins the second auction in the series of auctions from the first to the $m$ th one where

$$
\beta_{1}(2,1, m, n)=(1-1 / n)(1 /(n-1))=1 / n .
$$

This is because a bidder can win the second auction if he loses the first one this has probability $(1-1 / n)$. The probability of winning the second auction is $1 /(n-1)$. If he wins the second auction then he does not participate in the remaining auctions. In the same way, for $1 \leq y \leq m$, we get $\beta_{1}(y, 1, m, n)$ as:

$$
\begin{aligned}
\beta_{1}(y, 1, m, n) & =\frac{1}{n-y+1} \prod_{k=1}^{y-1}\left(1-\frac{1}{n-k+1}\right) \\
& =\frac{1}{n-y+1} \prod_{k=1}^{y-1} \frac{n-k}{n-k+1} \\
& =\frac{1}{n} .
\end{aligned}
$$


In general, for $j \leq y \leq m, \beta_{1}(j, y, m, n)$ is given by:

$$
\begin{aligned}
\beta_{1}(y, j, m, n) & =\frac{1}{n-y+1} \prod_{k=j}^{y-1}\left(1-\frac{1}{n-k+1}\right) \\
& =\frac{1}{n-y+1} \prod_{k=j}^{y-1} \frac{n-k}{n-k+1} \\
& =\frac{1}{n-j+1} .
\end{aligned}
$$

Note that $\beta_{1}(y, j, m, n)$ does not depend on $y$. Intuitively, before the beginning of the $j$ th auction, all bidders are symmetric with respect to winning the $y$ th auction, and there are $n-j+1$ bidders left at that point. Hence, each bidder's probability of winning the $y$ th auction is $1 /(n-j+1)$. The winner's expected profit for the $(y-1)$ th auction depends on this probability.

Let $\operatorname{EP}(j, m, n)$ denote the winner's expected profit for the $j$ th auction in the series of $m$ auctions with $n$ bidders for the first one. Likewise, let $\alpha(j, m, n)$ denote a bidder's ex-ante expected profit from winning any one auction in the series of auctions from the $j$ th (for $1 \leq j \leq m$ ) to the $m$ th one. This profit is:

$$
\begin{aligned}
\alpha(j, m, n) & =\sum_{y=j}^{m} \beta_{1}(y, j, m, n) E P(y, m, n) \\
& =\frac{1}{n-j+1} \sum_{y=j}^{m} E P(y, m, n) .
\end{aligned}
$$

A definition for $\operatorname{EP}(y, m, n)$ will be given in shortly. Note that since there are $m$ objects, $\alpha(m+1, m, n)=0$.

Given that the number of objects is $m$ and the number of bidders for the first auction is $n$, for auction $j, E S(j, m, n)$ denotes the expected surplus (surplus is what gets split between the auctioneer and the winning bidder, and it is synonymous with efficiency), and $E R(j, m, n)$ the expected revenue. Finally, for $n$ bidders, $E\left(f_{j}^{n}\right)$ and $E\left(s_{j}^{n}\right)$ denote the expected first and second order statistic [6] for the distribution $F$, from which the bidders draw their budget constraints for auction $j$.

We now find the equilibrium bids for second price rules in Section 4.1.

\subsection{Second price rules}

We now show that the equilibrium for SPSB rules are as follows. For auction $A_{j}$ $(1 \leq j \leq m)$ the equilibrium bid is:

$$
B_{j}(x)=\min \left\{x, \max \left\{0, a_{j}-\alpha(j+1, m, n)\right\}\right\}
$$

where $x$ is a bidder's budget constraint, $a_{j}$ is the common value of auction $A_{j}$, and $n$ is the number of bidders for the first auction.

In order to show this, we begin with the last auction and then reason backwards. Recall that a bidder comes to know its budget constraint for $A_{j}$ just before auction $j$ begins (i.e., after the previous $j-1$ auctions are over). 
Consider auction $m$. The number of bidders for this auction is $n-m+1$. Since this is the last auction, the bidding strategies for it are the same as those for a single object auction [20]. Hence we get the following:

$$
\begin{aligned}
& E P(m, m, n)=a_{m}-E\left(s_{m}^{n-m+1}\right) \\
& E R(m, m, n)=E\left(s_{m}^{n-m+1}\right) \\
& E S(m, m, n)=a_{m}
\end{aligned}
$$

Now consider auction $j(1 \leq j<m)$. Consider bidder 1 and suppose that $b^{*}$ is the highest competing bid. By bidding $T=a_{j}-\alpha(j+1, m, n)$, the bidder will win if $T>b^{*}$ and lose if $T<b^{*}$. Now suppose that he bids $z_{1}<T$. If $T>z_{1} \geq b^{*}$, then he still wins and his profit is still $T-b^{*}$. If $b^{*}>T>z_{1}$, he still loses. But, if $T>b^{*}>z_{1}$, then he loses whereas if he had bid $T$ he would have made a positive profit. Thus, bidding less than $T$ can never increase his profit, but in some cases it may actually decrease it. A similar argument shows that it is not profitable to bid more than $T$.

Note that, for auction $j, \alpha(j+1, m, n)$ is a bidder's expected ex-ante profit from winning a future auction and is therefore constant (i.e., it is the same for all the bidders). Now, this constant may be greater than $a_{j}$ or less than it. Let $C$ denote the condition $\alpha(j+1, m, n)>a_{j}$. We first analyze the case where $C$ is true and then the case where $C$ is false.

$C$ True: For this case, $a_{j}-\alpha(j+1, m, n)$ is negative, so the equilibrium bid for a bidder with budget $x$ is:

$$
\begin{aligned}
B_{j}(x) & =\min \left\{x, \max \left\{0, a_{j}-\alpha(j+1, m, n)\right\}\right\} \\
& =0
\end{aligned}
$$

Here, the bidder bids zero. The winner pays nothing, so the winner's profit, the surplus, and the revenue are:

$$
\begin{aligned}
& E P(j, m, n)=a_{j} \\
& E S(j, m, n)=E P(j, m, n) \\
& E R(j, m, n)=0
\end{aligned}
$$

$C$ False: For this case, $a_{j}-\alpha(j+1, m, n)$ is positive, so the equilibrium bids are:

$$
B_{j}(x)=\min \left\{x, a_{j}-\alpha(j+1, m, n)\right\}
$$

Here, the expected surplus, the expected revenue, and the winner's expected profit for auction $A_{j}$ depend on the relationship between $x$ and $a_{j}-\alpha(j+$ $1, m, n)$. Let the budget constraints of the $n$ bidders be $x_{n}>x_{n-1}>\cdots>$ $x_{1}$. Then, there are 3 cases we need to consider. These cases are as follows:

1. Case 1: $a_{j}-\alpha(j+1, m, n)>x_{n}$,

2. Case 2: $x_{n}>a_{j}-\alpha(j+1, m, n)>x_{n-1}$ and,

3. Case 3: $x_{n-1}>a_{j}-\alpha(j+1, m, n)$.

We now analyze each of these cases. In what follows, we let $\bar{n}$ denote the number of bidders for whom $x<a_{j}-\alpha(j+1, m, n)$. 
Consider Case 1. For this case, $\bar{n}=n$. Each bidder bids its own budget constraint. So the object gets allocated to the bidder with the highest budget. The winner pays the second highest budget, so the winner's profit (denoted $W P_{1}$ ), the surplus (denoted $W S_{1}$ ), and the revenue (denoted $W R_{1}$ ) are:

$$
\begin{aligned}
E P(j, m, n) & =a_{j}-E\left(s^{n-j+1} \mid f_{j}^{n-j+1}<a_{j}-\alpha(j+1, m, n)\right) \\
& =W P_{1} \\
E R(j, m, n) & =E\left(s^{n-j+1} \mid f_{j}^{n-j+1}<a_{j}-\alpha(j+1, m, n)\right) \\
& =W R_{1} \\
E S(j, m, n) & =a_{j}=W S_{1}
\end{aligned}
$$

Consider Case 2. For this case, $\bar{n}=n-1$, i.e., for only one bidder $x>a_{j}-$ $\alpha(j+1, m, n)$ while the remaining $n-1$ bidders $x<a_{j}-\alpha(j+1, m, n)$. Thus, only one bidder bids $a_{j}-\alpha(j+1, m, n)$, while the remaining $n-1$ bidders bid their respective budget constraint. Here the object is allocated to the highest bidder, i.e., the one which bids $a_{j}-\alpha(j+1, m, n)$. The winner pays the second highest bid. So the winner's profit (denoted $W P_{2}$ ), the surplus (denoted $W S_{2}$ ), and the revenue (denoted $W R_{2}$ ) are:

$$
\begin{aligned}
E P(j, m, n)= & a_{j}-E\left(s_{j}^{n-j+1} \mid\right. \\
& \left.f_{j}^{n-j+1}>\alpha(j+1, m, n)>s_{j}^{n-j+1}\right) \\
= & W P_{2} \\
E R(j, m, n)= & E\left(s_{j}^{n-j+1} \mid\right. \\
& \left.f_{j}^{n-j+1}>\alpha(j+1, m, n)>s_{j}^{n-j+1}\right) \\
= & =W R_{2} \\
E S(j, m, n)= & a_{j}=W S_{2}
\end{aligned}
$$

Consider Case 3 . For this case, $\bar{n} \leq n-2$, i.e., two bidder bid $a_{j}-\alpha(j+$ $1, m, n)$ while the remaining $n-2$ bidders bid their respective budget constraint. There are two highest bids (each $a_{j}-\alpha(j+1, m, n)$ ) and the object is allocated to one of these with equal probability. The winner pays the second highest bid (i.e., $a_{j}-\alpha(j+1, m, n)$ ). So the winner's profit (denoted $W P_{3}$ ), the surplus (denoted $W S_{3}$ ), and the revenue (denoted $W R_{3}$ ) are:

$$
\begin{aligned}
& E P(j, m, n)=0.5 \times \alpha(j+1, m, n)=W P_{3} \\
& E R(j, m, n)=a_{j}-\alpha(j+1, m, n)=W R_{3} \\
& E S(j, m, n)=a_{j}=W S_{3}
\end{aligned}
$$

By combining these three cases, we get:

$$
\begin{aligned}
& E P(j, m, n)=P_{1} \times W P_{1}+P_{2} \times W P_{2}+P_{3} \times W P_{3} \\
& E S(j, m, n)=P_{1} \times W R_{2}+P_{2} \times W R_{2}+P_{3} \times W R_{3} \\
& E R(j, m, n)=P_{1} \times W S_{2}+P_{2} \times W S_{2}+P_{3} \times W S_{3}
\end{aligned}
$$

where $P_{1}, P_{2}$, and $P_{3}$ denote the probability of occurence of Case1, Case2, and Case 3 respectively. These probabilities are:

$$
\begin{aligned}
& P_{1}=\left[F\left(a_{j}-\alpha(j+1, m, n)\right)\right]^{n} \\
& P_{2}=\left[1-F\left(a_{j}-\alpha(j+1, m, n)\right)\right] \times\left[F\left(a_{j}-\alpha(j+1, m, n)\right)\right]^{n-1} \\
& P_{3}=1-P_{1}-P_{2}
\end{aligned}
$$


Given $\alpha(j+1, m, n)$, we find $E P(j, m, n)$ and given $\alpha(y, m, n)$ for $j+1 \leq$ $y \leq m$, we find $\alpha(j, m, n)$ using Equation 5.

In the same way, it can be shown that the equilibrium bids for English auction rules are the same a those given in Equation 6.

\section{Related Work}

To date, most of the work on auctions for multiple heterogeneous objects has focused on combinatorial auctions $[19,18]$. There has been relatively little work on sequential auctions. Furthermore, most of the work in this area focuses on scenarios in which all objects are identical [21, 22, 12, 15]. For example, Vickrey [21], studies auctions for multiple identical objects. In this model $n$ identical objects are bid for at the same time and an object is awarded to each of the $n$ highest bidders. Milgrom [15] studies auctioning of multiple identical objects in a series of sequential auctions where only one object is sold at each auction. This private-values model compares revenues for first- and second-price sequential auctions in which no bidder requires more than one object. While [21, 15] assume that all buyers have single unit demand, [22, 12] study sequential auctions with multi-unit (i.e., two unit) demand. Also, [12] finds symmetric equilibrium for a two-stage secondprice auction and [22] finds equilibrium for a two-unit pay-your-bid-auction. Our work differs from the above in that we deal with multiple heterogeneous objects.

Auctions for multiple heterogeneous objects have been studied in [4]. This work is similar to ours in the sense that it deals with heterogeneous objects. However, while our work focuses on sequential auctions, [4] studies simultaneous auctions for multiple objects. Multi-object auctions have also been studied in [5, 1] - this work determines efficient bidding strategies for heterogeneous private-value objects. Our work differs in the following way. We focus on common-value auctions for bidders that have budget-constraints.

Sequential auctions for heterogeneous private-value objects have been studied in [16], and those for common-value objects in [2], but both of them under the assumption of complete information. In contrast to this, our work focuses on an incomplete information setting with budget constraints. Elmaghraby [8] studies sequential auctions for heterogeneous private-value objects using only the secondprice sealed-bid rules. Fatima et al $[10,9,11]$ studied sequential auctions for common and private value objects using English auction rules and SPSB rules for bidders without budget constraints. Comparing $[10,9,11]$ with this paper, we see that the bidding strategies for the former are special cases of the latter. In a similar scenario, [7] considers sequential auctions for private value objects. This paper differs from $[7,8,10,9,11]$ in that, unlike others, we focus on common-value auctions for budget-constrained bidders in an incomplete information setting.

\section{Conclusions and Future Work}

This paper analyzes sequential auctions for budget constrained bidders, for multiple heterogeneous common value objects. In such auctions, the bidders' problem is to determine how much to bid at each auction. In this paper we showed that the strategic bidding behavior depends on the agenda; if we change the agenda, then the equilibrium bids also change. Then for a given agenda, we determined 
equilibrium bids for each individual auction in a series. We did this for both firstand second-price rules in an incomplete information setting where the bidders are uncertain about the budget constraints. Comparing $[10,9,11]$ with this paper, we see that the bidding strategies for the former are special cases of the latter.

The bidding strategies thus determined are useful for developing agents that bid on our behalf. There are several interesting directions for future work. First, this paper studied sequential auctions where each bidder has unit demand. In future, it would be interesting to extend this analysis to the case where a bidder needs more than one object. Second, we treated the values of the objects as common knowledge. However, in some real-world scenarios, the players may not know the value of the objects but may have only probabilistic information about the values of the objects. It is therefore important to extend the current analysis by treating the values of objects as probabilistic information.

\section{References}

[1] P. Anthony and N. R. Jennings. Developing a bidding agent for multiple heterogeneous auctions. ACM Transactions on Internet Technology, 3(3):185217, 2003.

[2] J. P. Benoit and V. Krishna. Multiple-object auctions with budget constrained bidders. Review of Economic Studies, 68:155-179, 2001.

[3] D. Bernhardt and D. Scoones. A note on sequential auctions. American Economic Review, 84(3):653-657, 1994.

[4] S. Bikhchandani. Auctions of heterogeneous objects. Games and Economic Behavior, 26:193-220, 1999.

[5] A. Byde, C. Preist, and N. R. Jennings. Decision procedures for multiple auctions. In International Conference on Autonomous Agents and MultiAgent Systems, pages 613-620, Bologna, Italy, 2002.

[6] H. David. Order Statistics. Wiley, New York, 1969.

[7] E. Elkind and S. Fatima. Maximizing revenue in sequential auctions. In LNCS Vol 4858, pages 491-502, 2007.

[8] W. Elmaghraby. The importance of ordering in sequential auctions. Management Science, 49(5):673-682, 2003.

[9] S. S. Fatima. Sequential versus simultaneous auctions: A case study. In Eighth International Conference on Electronic Commerce, pages 82-91, Fredericton, Canada, 2006.

[10] S. S. Fatima, M. Wooldridge, and N. R. Jennings. Sequential auctions for objects with common and private values. In Fourth International Conference on Autonomous Agents and Multi-Agent Systems, pages 635-642, Utrecht, Netherlands, 2005.

[11] S. S. Fatima, M. Wooldridge, and N. R. Jennings. Sequential auctions in uncertain information settings. In Ninth International Workshop on AgentMediated Electronic Commerce, pages 15-28, Honolulu, Hawaii, 2007.

[12] B. Katzman. A two stage sequential auction with multi-unit demands. Journal of Economic Theory, 86:77-99, 1999. 
[13] S. Kraus. Strategic negotiation in multi-agent environments. The MIT Press, Cambridge, Massachusetts, 2001.

[14] P. Milgrom and R. J. Weber. A theory of auctions and competitive bidding. Econometrica, 50(5):1089-1122, 1982.

[15] P. Milgrom and R. J. Weber. A theory of auctions and competitive bidding II. In The Economic Theory of Auctions. Edward Elgar, Cheltenham, U.K, 2000.

[16] C. Pitchik and A. Schotter. Perfect equilibria in budget-constrained sequential auctions: An experimental study. Rand Journal of Economics, 19:363388, 1988.

[17] J. S. Rosenschein and G. Zlotkin. Rules of Encounter. MIT Press, 1994.

[18] T. Sandholm and S. Suri. BOB: Improved winner determination in combinatorial auctions and generalizations. Artificial Intelligence, 145:33-58, 2003.

[19] T. Sandholm, S. Suri, A. Gilpin, and D. Levine. CABOB: A fast optimal algorithm for combinatorial auctions. In Proceedings of the International Joint Conference on Artificial Intelligence (IJCAI-01), pages 1102-1108, Seattle, Washington, 2001.

[20] W. Vickrey. Counterspeculation, auctions and competitive sealed tenders. Journal of Finance, 16:8-37, 1961.

[21] W. Vickrey. Auctions and bidding games. Recent Advances in Game Theory, 29:15-27, 1962.

[22] R. E. Wiggans and C. M. Kahn. Multi-unit pay your-bid auctions with variable rewards. Games and Economic Behavior, 23:25-42, 1998. 